\title{
STUDY OF POLYMORPHISMS ASSOCIATED TO HIRSCHSPRUNG DISEASE IN SPANISH POPULATION
}

L. Vega Hernández ${ }^{1}$, L. Castaño González ${ }^{1,2}$, E. Ruíz Aja ${ }^{3}$, N. Martínez Ezquerra ${ }^{4}$, E. de Diego García ${ }^{5}$, P. López Álvarez-Buhilla ${ }^{3}$

${ }^{1}$ Research Unit, Cruces Hospital, Barakaldo, ${ }^{2}$ University of Basque Country (UPV-EHU), Leioa, ${ }^{3}$ Pediatric Surgery, ${ }^{4}$ Pediatrics, Cruces Hospital, Barakaldo, ${ }^{5}$ Pediatric Surgery, Marqués de Valdecilla University Hospital, Santander, Spain

Background and aims: Hirschsprung disease (HSCR) is a congenital disorder characterized by the absence of ganglion cells along the gastrointestinal tract. It is caused by defects in the migration of enteric nervous system cells during the embryonic development. Improved surgical treatments have reduced the mortality of the patients, which facilitates the genetic study of patients and their families. The inheritance pattern of this disease is still unknown, but the RET proto-oncogene is the major gene involved. There are several mutations and polymorphisms (SNPs) described in RET as associated to the disease, suggesting a genetic predisposition in the HSCR population. Our goal is to find and analyze these disease-associated SNPs and establish a genetic model.

Methods: We have analyzed the promoter and several exons of RET in different populations: HSCR patients, healthy subjects and patients with medullary thyroid carcinoma (CMT, also caused by mutations in RET) by PCR and sequencing.

To find out if some SNPs previously described as associated with HSCR are also present in our population, we analyzed them by TaqMan ${ }^{\circledR}$ probes.

Results: The TaqMan ${ }^{\circledR}$ results reveals that RET alleles T, G and C of SNPs p.L769L (exon13), p.G691S (Exon11) and p.S904S (exon15) respectively, are associated with our HSCR population. There are also a region in RET promoter where there are several different SNPs. The differences are significant compared with healthy people or CMT patients.

Conclusions: The presence of certain polymorphisms in the RET proto-oncogene in spanish population suggests a genetic predisposition to suffer Hirschsprung disease. 